\title{
MICROSEGURO FAMILIAR PARA ALIMENTACIÓN, MEDICAMENTOS Y APOYO EDUCATIVO: UN PRODUCTO CON VALOR DIFERENCIAL EN CASO DE DESEMPLEO*
}

\section{FAMILY MICROINSURANCE FOR FOOD, MEDICINES AND EDUCATIONAL SUPPORT: A PRODUCT WITH A DIFFERENTIAL VALUE IN CASE OF UNEMPLOYMENT}

\author{
JOHN EDISSON URREGO ROMERO** \\ GLADYS YANETH BELTRÁN PAIPA ${ }^{* * *}$ \\ MANUEL ALBERTO GRANADOS DÍAZ ${ }^{* * * * *}$
}

Fecha de recepción: 14 de agosto de 2017

Fecha de aceptación: 4 de abril de 2018

Disponible en linea: 30 de junio de 2018

\section{Para Citar este articulo/To cite this article}

Urrego Romero. John Edisson, Beltrán Paipa, Gladys Yaneth \& Granados Díaz, Manuel Alberto, Microseguro familiar para alimentación, medicamentos y apoyo educativo: un producto con valor diferencial en caso de desempleo, 48 Rev.Ibero-Latinoam.Seguros, 147-160 (2018). https://doi.org/10.11144/Javeriana.ris48.mfam

doi:10.11144/Javeriana.ris48.mfam

* El artículo es resultado de la investigación "Diseño de un MS de aporte legal en salud y alimentación" adelantada en el Grupo de Investigación, desarrollo e innovación del Sector Financiero GIDISF del SENA Centro de Servicios financieros de Bogotá que se realizó entre los años 2015 y 2016.

** Candidato a Magister en Sociología de la Universidad Nacional de Colombia. Licenciado en Filosofía con énfasis en pensamiento político y económico de la Universidad Santo Tomás. Instructor / Investigador SENA. Miembro del Grupo GIDiSF. Contacto: jeurrego77@misena.edu.co

*** Especializada en Administración de Riesgos de la Institución Universitaria Politécnico Grancolombiano. Administradora de Empresas. Instructora/Investigadora SENA. Miembro del grupo GIDiSF. Contacto: gyaneth60@misena.edu.co

**** Especialista en Finanzas de la Universidad de los Andes y Actuaría de la Universidad Antonio Nariño. Estadístico de la Universidad Nacional de Colombia. Docente de actuaría en la Universidad Nacional de Colombia, Universidad Antonio Nariño y el Instituto Nacional de Seguros. Contacto: albertogranadosd@hotmail.com 


\section{RESUMEN}

El microseguro (MS) es un producto de protección a personas en condiciones de vulnerabilidad que brinda alternativas para mitigar el riesgo. El artículo quiere dar cuenta de la innovación que tiene un MS familiar en caso de desempleo al cubrir alimentación y brindar un apoyo para educación y medicamentos por 6 meses en poblaciones vulnerables al desempleo de los estratos 1, 2 y 3 de la ciudad de Bogotá. La metodología empleada fue un estudio de mercado que permitió establecer las condiciones desfavorables a nivel de empleabilidad formal e informal, alimentación y bienestar social en 3 barrios de Bogotá para establecer las coberturas y condiciones que debe tener la póliza del MS. Este proceso de realizó a través de un modelo actuarial que permitió calcular las Tasas Puras de Riesgo Mensuales TPRD(m) y TPRIT(m), Tasas Comerciales Mensuales TCD (m) y TCIT (m) para determinar las Primas Comerciales Mensuales PCD (m) y PCIT (m) de las coberturas para desempleo involuntario e incapacidad temporal. De acuerdo con los resultados obtenidos se valida la disponibilidad que tienen estas poblaciones para adquirir el MS que se caracteriza por su prima baja, cobertura de alimentación con un $20 \%$ en apoyo educativo y $20 \%$ de medicamentos sobre alguna de las tres alternativas de valor asegurado que puede tomar voluntariamente el consumidor para cumplir con la funcionalidad de mitigar los riesgos alimentarios y proteger el desarrollo familiar y social de cada una de estas poblaciones.

Palabras clave: Microseguro; riesgo alimentario; desempleo; población vulnerable. 


\begin{abstract}
Microinsurance (MS) is a product of protection to people in conditions of vulnerability that provides alternatives to mitigate the risk. The article wants to give an account of the innovation that a family MS has in the event of unemployment by covering food and providing support for education and medication for 6 months in populations vulnerable to unemployment in strata 1, 2 and 3 of the city of Bogota. The methodology used was a market study that allowed to establish the unfavorable conditions a level of formal and informal employability, food and social welfare in 3 neighborhoods of Bogotá to establish the coverages and conditions that should have the MS policy. This process of realization through an actuarial model that allowed it to calculate the TPRD (m) and TPRIT (m), Monthly Commercial Rates TCD (m) and TCIT (m) to calculate the Monthly Trade Premiums PCD) And PCIT (m) of the coverages for involuntary unemployment and temporary incapacity. According to the results obtained, the availability of the populations to acquire MS that are characterized by their low premium, food coverage with $20 \%$ in educational support and $20 \%$ of the medicines on one of the three alternatives of insured value that can be taken by the user to comply with the functionality of mitigating food risks and protecting the family and social development of each of these populations.
\end{abstract}

Keywords: Microinsurance; food risk; vulnerable population; unemployment.

\title{
SUMARIO
}

1. INTRODUCCIÓN. 2. EL MICROSEGURO A NIVEL MUNDIAL. 3. MS EN AMÉRICA LATINA. 4. EL MS EN COLOMBIA. 5. MÉTODO. 6. RESULTADOS. 6.1. Generalidades del estudio de mercado. 6.2. Descripción del MS familiar para alimentación con apoyo educativo y medicamentos. 6.3. Aspectos técnicos, administrativos y financieros. 7. CONCLUSIONES. BIBLIOGRAFÍA 


\section{INTRODUCCIÓN}

El microseguro (MS) es un producto financiero que cumple una función inclusiva al brindar la oportunidad de protección a las poblaciones más pobres contra algunos riesgos producidos por la naturaleza o aquellos que afectan a las personas y sus bienes ${ }^{1}$. Los MS se caracterizan por ser productos especializados en mitigar riesgos presentes en comunidades vulnerables, cuyas condiciones y procedimientos deben pensarse desde la simplicidad y el fácil acceso reflejados en las primas asequibles a poblaciones pobres con acceso a través de canales de comercialización que están a la mano de los consumidores (Camargo, Tatin-Jalerán y Furst 2014).

Según Churchill y Matul (2014), el MS se define como la protección de la población de bajos ingresos que mitiga determinados riesgos por pagos regulares de primas proporcionales a la posibilidad y costo del riesgo envuelto. Este concepto ha sido asumido por el sector asegurador que reafirma la funcionalidad del MS y plantea nuevos retos para promover la cultura del seguro que incentive los pagos de primas de forma regular para minimizar el riesgo al que se pueden ver expuestos.

Por otro lado, los canales de distribución de estos productos deben ser adecuados para la población de bajos ingresos que permitan el uso y la cobertura no sólo al momento de tomarlo, sino para el recaudo de las primas que se caracterizan por ser mensuales o bimestrales, igual o inferior a la doceava parte de 1 Salario Mínimo Mensual Legal Vigente (SMMLV). Las facilidades para el acceso del canal es la alternativa dada a los consumidores que no sólo les permite acceder al producto, sino que le facilita el medio de pago para tomar el MS.

El artículo describe las características y condiciones del MS familiar que cubre alimentación y brinda un apoyo para educación y medicamentos en caso de desempleo. Los datos utilizados para el diseño se

1 Los riesgos de la naturaleza son (desastres naturales como terremotos, inundaciones, maremotos, sequías entre otros). Los riesgos personales son los que amenazan la integridad física o corporal de la persona (enfermedad, muerte, desempleo, accidentes, entre otros). Los riesgos de los bienes son los que afectan la integridad de las cosas corporales (incendio, inundación, hurto, terremoto, avería entre otros). 
tomaron de una encuesta aplicada en 3 barrios de Bogotá que fueron convalidados con los datos generales de la encuesta nacional de hogares 2015 emitidos por el DANE. La medición estadística se determinó en las variables de nivel de consumo, nivel de ingresos, hábitos de ahorro, disponibilidad para adquirir el MS por estrato socioeconómico², para establecer los rangos de consumo y el rango de la prima según la temporalidad de los amparos, modalidad del seguro, y las medidas de control como suscripción, riesgo y periodos de carencia (Beltrán y Urrego 2015).

Los resultados muestran la factibilidad que tiene el desarrollo de un MS enfocado a cubrir alimentación y brindar apoyos a medicamentos y educación en función de las necesidades mínimas vitales que tienen estas poblaciones vulnerables. La prima es una posibilidad de fácil acceso para los estratos 1, 2 y 3 por su cuantía y su impacto se evidencia al momento de hacer el pago del seguro en condiciones de desempleo. De ahí, que el MS sea un producto inclusivo para un nicho de mercado, convirtiéndose en una oportunidad que posibilita la sostenibilidad económica y la prevención de riesgo en su ingesta alimentaria.

\section{EL MICROSEGURO A NIVEL MUNDIAL}

Los primeros indicios de venta de seguros pequeños se remontan a Nueva York en 1868 cuando se inicia la venta de seguros de vida en las puertas de las fábricas de las grandes ciudades de Estados Unidos, por intermedio de la Metropolitan Life Insurance Company que era la aseguradora más grande del mundo y cuyos productos fueron la base para los distintos mercados de la actualidad. La comercialización de los seguros de vida se realizaba por medio de agentes que vendían puerta a puerta, con un recaudo de las cuotas según el riesgo de los trabajadores de la industria en los días del pago laboral y unas coberturas que eran acordes con las necesidades de los trabajadores.

2 La estratificación socioeconómica es una clasificación de la población en seis niveles de acuerdo con el grado de riqueza y calidad de vida de los hogares. Los estratos son determinados indirectamente por las condiciones físicas de las viviendas y su localización según el DANE. El estrato uno hace referencia a los hogares con menor riqueza y calidad de vida $\mathrm{y}$, el seis a los hogares de mejores condiciones socioeconómicas (Beltrán y Urrego, 2015). 
Hace aproximadamente 35 años el economista bangladés Muhammad Yunus $^{3}$ creó el Banco de los pobres (Grameen Bank) mediante una propuesta prestamista de pequeñas sumas de dinero a las mujeres de Jobra, un pueblo de Blangladés azotado por las inundaciones. Se estableció un producto llamado microcrédito, un producto de crédito que buscaba potenciar la creación y el desarrollo de negocios para las mujeres, la educación de sus hijos, la compra de vivienda y los gastos generales basados en la confianza mutua, la responsabilidad y la participación (Mena 2004). Con el fin de respaldar esta deuda crediticia fue necesario que el prestatario tomara un MS, pues en caso de fallecimiento de éste, la aseguradora pagaría la deuda al banco y los familiares no quedarían endeudados. En este orden de ideas, el MS nace ligado al microcrédito como garantía en el riesgo de pago por parte de los deudores. En este marco, los MS están asociados a los productos de vida, exequias y propiedad, como requisito para la concesión del microcrédito.

\section{MS EN AMÉRICA LATINA}

La participación de los MS en América Latina y el Caribe se enmarca en un total de 44,9 millones de personas y sus propiedades. Mediante un estudio del Fondo Multilateral de Inversiones por McCord, Ingram y Tatin-Jaleran (2014) se determinó que los MS se encuentran en 19 de los 20 países de América Latina y el Caribe y más del 55\% o 25,1 millones de personas con coberturas de MS se encuentran en dos países: México y Brasil. Por otra parte, alrededor de1 90\% de las vidas y bienes amparados en América Latina y el Caribe se encuentran en los cinco países con mayor número de asegurados - México, Brasil, Colombia, Perú y Ecuador.

El número total de asegurados en los países estudiados ha crecido 4,2 veces desde 2005 con un total de los 44,9 millones de personas, mostrando un incremento importante el MS de accidentes personales,

3 Muhammad Yunus nació en Bangladesh en 1940. Es doctor en economía y muy famoso por ser el creador de los microcréditos y del Banco de los Pobres. Ha luchado de forma incansable por la disminución de la pobreza de Bangladesh lo que le concedió el galardón del Premio Nobel de la Paz en octubre de 2006. El Proyecto Grameen Bank nació en Jobra, una aldea de Bangladesh en 1976. En 1983 se convirtió en un banco formal en virtud de una ley especial aprobada para su creación. Es propiedad de los prestatarios pobres del banco, que en su mayoría son mujeres. Esta entidad trabaja exclusivamente para ellos. Los prestatarios del Grameen Bank poseen en la actualidad el 94\% del capital total del banco. El 6\% restante es propiedad del gobierno. (Yunus 2008). 
seguido por el de salud y propiedad. Según los estudios el 71\% están cubiertos por un MS de vida; el 34\% cuenta con un producto de vida deudores el cual respalda el monto de una deuda en caso de fallecimiento del deudor; el MS de salud es la siguiente cobertura en importancia con el $22 \%$ del total de personas aseguradas, finalmente el $6 \%$ cuenta con un MS de propiedad.

En América Latina y el Caribe se aprecia una alta concentración de compañías de seguros comerciales que lideran el mercado de los MS y algunas de éstas advierten que tratan de hacer productos de MS a la medida de sus clientes, mientras que más de la mitad indican que adaptan sus operaciones para cumplir con las exigencias del MS. La situación anterior muestra que se requiere diseñar productos adecuados a las necesidades de los clientes y que las aseguradoras deben adaptar su estructura a operaciones de productos de MS, actitud que no se ha evidenciado porque más de la mitad de estas empresas reconoció no haber realizado ningún ajuste para promover el desarrollo y oferta de MS.

La contribución de las primas de MS al total de primas de una aseguradora es casi un 20\%, indicando que el MS se ha convertido en una herramienta clave en las operaciones de seguros, destacándose las Instituciones Microfinancieras (IMF), que son los canales a través de los cuales se comercializan la mayoría de productos de MS en la región. Por su parte México, Perú y Colombia son los países con mayor diversidad de canales de distribución a nivel regional. Según el Panorama de los Microseguros en América Latina y el Caribe ${ }^{4}$, la gran preocupación de los proveedores de MS es cómo llegar a la población de bajos ingresos, con productos ajustados a sus necesidades manteniendo un proceso simple y económico

\section{EL MS EN COLOMBIA}

Durante los últimos años, en Colombia se han comercializado productos de MS en la línea de los seguros masivos de bajo costo; sin embargo, estos

4 El "Panorama de los Microseguros en América Latina y el Caribe" fue comisionado por el Fondo Multilateral de Inversiones (FOMIN), un miembro del Banco Interamericano de Desarrollo (BID), y fue desarrollado por el MicroInsurance Centre. El estudio recibió la colaboración de la CitiFoundation y de la Munich Re Foundation. 
no fueron diseñados teniendo en cuenta las necesidades y características de la población más pobre, razón por la cual se hace necesario reorientarlos a cumplir con la naturaleza de mitigar riesgos de poblaciones en condiciones de vulnerabilidad, cumplir estándares de coberturas claras y sencillas, fácil y económico pago de primas, rapidez en la expedición y trámites de reclamaciones de una forma ágil y oportuna para evitar confusiones al momento de tomarlo.

Así las cosas, se dificulta establecer con claridad cuál ha sido la evolución del mercado de MS, ya que gran parte de productos de seguros se catalogan como MS por el mercado asegurador, cuando realmente son productos masivos de bajo costo, situación que ratifica Fasecolda por cuanto las compañías de seguros le reportan datos de forma general e indiscriminada y no especificando los valores de primas correspondientes a productos de MS y de seguros masivos.

Si bien los MS se destacan por ser una oferta de productos voluntarios y no obligatorios, algunos puntos negativos que influyen en el desarrollo de estos productos son: la falta de estudios de demanda, poca diversificación de productos, altos costos de distribución, desaprovechar canales de distribución con potencial para llegar a estratos 1, 2 y 3, dificultad en el cobro de primas, procedimientos complicados, la póliza redactada en términos complejos que carece de simplicidad (Camargo et al, 2014).

Cabe resaltar el interés del gobierno colombiano durante los últimos años en el desarrollo de productos de MS, mediante la inclusión financiera, educación financiera, gestión de riesgos, a través de alianzas públicoprivadas (APP) para que los estratos 1 y 2 tengan acceso al seguro, lo que genera aprendizaje y mayor nivel de confianza y conocimiento, a pesar de la insuficiencia en la difusión y el uso de la información.

\section{MÉTODO}

Se trata de un estudio actuarial que parte de un modelo estadísticomatemático para evaluar el impacto financiero del riesgo en el diseño del MS familiar, el cual busca garantizar una cobertura de alimentación con apoyo educativo y de medicamentos a la población objetivo. En un primer momento se analizaron los datos tomados de la encuesta aplicada a 3 
barrios de Bogotá para identificar los riesgos que afectan el sostenimiento de los hogares, la cultura de ahorro y opciones de supervivencia en caso de desempleo de cada una de las poblaciones; todas ellas en relación con la disponibilidad para adquirir dicho producto. La metodología utilizada fue la del estudio de mercado a través de una encuesta semiestructurada de demanda de necesidades para el desarrollo de MS que se aplicó a 300 personas ubicadas en tres barrios de Bogotá: Caracolí de la Localidad Ciudad Bolívar (estrato 1), El Recreo de la Localidad de Bosa (estrato 2) y Alquería de la Localidad de Puente Aranda (estrato 3$)^{5}$.

Por otro lado, los datos se tomaron las estadísticas del DANE sobre desempleo y población activa, así como de la Tabla Colombiana de Mortalidad de Asegurados con los que se determinaron las variables socioeconómicas medidas para la estimación de los porcentajes de Tasa de riesgo-muerte, Tasa de Riesgo Invalidez, Tasa Comercial de Invalidez tanto de hombres (qx) como mujeres (qy) para la cobertura de incapacidad por invalidez, lo que lleva a establecer la Tasa de Invalidez Ponderada (qz). Para la cobertura de desempleo se determinaron la frecuencia, la severidad y la Tasa Comercial Anual, la Tasa Comercial Mensual de desempleo que se toma de una Tasa Pura Anual de riesgo de desempleo por estrato. Este promedio se tomó del ponderado de tasas de desempleo por estrato entre los años 2012 y 2015.

La metodología actuarial para calcular las Tasas Puras de Riesgo Mensuales para las Coberturas de Desempleo Involuntario para el Trabajador Independiente, TPRD $(\mathrm{m})$ y de Incapacidad Temporal por Accidente o Enfermedad para el Trabajador Independiente ó Informal, TPRIT(m) es la siguiente:

En primer lugar, las Tasas Puras de Riesgo Mensuales para las Coberturas de Desempleo Involuntario para el Trabajador Independiente TPRD(m), se calcularon con base en TPRD(a): Tasa Pura de Riesgo Anual para la cobertura de Desempleo Involuntario donde $\operatorname{TPRD}(\mathrm{a})=$ FD(a) * SD siendo FD(a) la Variabilidad Promedio Anual Ajustada de la Tasa de Desempleo de Estratos 1, 2 y 3, ع: Factor de Seguridad, SD:

5 Para profundizar en los datos y las caracterizaciones se puede revisar el artículo sobre el estudio de mercado realizado durante el año 2015 (Beltrán y Urrego 2015). 
Severidad Esperada (Valor Asegurado), $\mathrm{SD}=6 / \mathrm{a} 6 \mid \mathrm{i}(12) * \mathrm{R}$ donde $\mathrm{R}$ es el Valor Asegurado Mensual y 6/a6|i(12) valor actual de una renta cierta de $\$ 1$ diferida 6 meses a una tasa mensual del i(12)\%, pagadera durante 6 meses; siendo $\mathrm{i}(12)=(1+\mathrm{i})^{(1 / 12)}-1$ donde $\mathrm{i}$ es la Tasa de Interés Técnico anual, es decir, i(12): Tasa de interés Técnico mensual. En resumen, la Tasa Pura de Riesgo Mensual para la cobertura de Desempleo Involuntario, está dada por:

$$
\operatorname{TPRD}(\mathrm{m})=(\operatorname{FD}(\mathrm{a}) / 12) *(1+\varepsilon){ }_{6 /} \mathrm{a}_{6 \mathrm{il}(12)}
$$

Por otro lado, la Tasa Pura de Riesgo Mensual para la cobertura de Incapacidad Temporal por Accidente o Enfermedad TPRIT(m) se calculó con base en la TPRIT(a): Tasa Pura de Riesgo Anual para la cobertura de Desempleo Involuntario TPRIT(a) que resulta de la FD(a) Frecuencia Anual de Siniestralidad donde $\operatorname{FD}(\mathrm{a})=(\mathrm{qz}) * \mathrm{v}^{*}(1+\gamma)$ siendo qz la probabilidad ponderada de fallecimiento asignada para el grupo asegurado, para un grupo con edad promedio estimada (z).

$$
\operatorname{TPRIT}(\mathrm{m})=\left(\left(\mathrm{q}_{\mathrm{z}}\right) * \mathrm{v}_{(\mathrm{m})} *(1+\gamma)\right){ }_{6 /} \mathrm{a}_{6 \mathrm{ji}(12)}
$$

La Tasa Pura de Riesgo Mensual para la Cobertura de Incapacidad Temporal por Accidente o Enfermedad, está dada siendo ${ }_{6 /} \mathrm{a}_{6 \mathrm{ii}(12)}$ el valor actual de una renta cierta de $\$ 1$ diferida 6 meses a una tasa mensual del $\mathrm{i}(12) \%, \mathrm{i}(12)=(1+\mathrm{i})^{(1 / 12)}-1$; i: Tasa de Interés Técnico; $\gamma$ : Factor de Seguridad; $v$ : Factor de descuento anual y v(m): Factor de descuento mensual.

\section{RESULTADOS}

\subsection{Generalidades del estudio de mercado}

Los niveles de desempleo se confrontan con los porcentajes de informalidad laboral que se presentan en Colombia. Según las estadísticas del DANE, las tasas de desempleo entre 2012 y 2015 tuvieron una disminución en todos los estratos socioeconómicos del país. De acuerdo con los datos, la varianza absoluta promedio en la tasa de desempleo entre 2012 y 2015 por estrato fueron 0,0328 para el estrato 1 (bajo-bajo), 0.0257 para el estrato 2 (bajo) y 0,0271 para el estrato 3 (medio bajo). Esto permitió determinar la tasa pura de riesgo anual para los estratos 1, 
2 y 3 que se estableció en 0.0383 . Las variaciones no son significativas por año, lo que permite una prospectiva del estudio de mercado para la implementación del MS sin grandes márgenes de error.

Por otro lado, el comportamiento de los tomadores en el mercado asegurador para ofrecer una sola tarifa en la cobertura de incapacidad temporal por accidente o enfermedad confirma que la población que tomaría el seguro sería del $60 \%$ hombres y $40 \%$ mujeres con una edad promedio de 38 años. Para el caso del amparo de enfermedad temporal se tuvieron en cuenta las tasas mensuales de incapacidad temporal por edad de la tarifación la Tabla Colombiana de Mortalidad de Asegurados por Género, TCM 98-03 emitida por Fasecolda para el análisis y medición de los niveles de probabilidad a nivel de incapacidad y siniestralidad de los futuros tomadores del MS con el fin de cuantificar el producto en función de los factores de seguridad y gastos administrativos que se incurren con la puesta del MS en el mercado asegurador.

Las estadísticas y datos tomados de una muestra de pobladores en la ciudad de Bogotá muestran un nivel favorable para que pueda ser tomado el MS en caso de desempleo; sin embargo, no son garantía de que pueda ser usado ya que es necesario estimarse después de al menos dos (2) años de comercialización del MS. El impacto será significativo en la medida que se promuevan estrategias de educación financiera, se realice una venta veraz del MS y se realice divulgación sobre las bondades que puede tener para personas con niveles altos de vulnerabilidad al quedar desempleadas o contraer enfermedad en el sector informal.

\subsection{Descripción del MS familiar para alimentación con apoyo educativo y medicamentos}

El MS para alimentación con apoyo educativo y medicamentos tiene un amparo básico que es alimentación y 2 amparos adicionales de apoyo educativo y apoyo para medicamentos. La modalidad del seguro es individual y se tomó la suscripción como la medida de control, el nivel de riesgo y el periodo de carencia que es de 6 meses continuos. La tarifa tiene tres tipos de montos asegurables para su escogencia y será provisional hasta después de 2 años según los niveles de siniestralidad reportados. 
Los eventos asegurables del MS son despido sin justa causa para un trabajador dependiente o incapacidad temporal por accidente o enfermedad para el trabajador independiente o informal. Dentro de las exclusiones se encuentra que el MS no cubrirá indemnización alguna, ni la compañía aseguradora estará obligada a hacerlo. El asegurado debe tomar el MS por 6 meses continuos antes de poder cobrarlo.

El cobro del seguro bajo la cobertura de desempleo involuntario, debe realizarlo una persona natural que cumpla con la edad de ser mayor de (18) años, encontrarse empleado bajo la legislación laboral mediante contrato laboral de trabajo a término indefinido o después de llevar dos (2) años continuos laborando bajo contrato a término fijo, no tener más de 56 años las mujeres y 61 años los hombres, con edades de permanencia hasta los 57 años para las mujeres y 62 años para los hombres.

En el caso de la cobertura de incapacidad temporal por accidente o por enfermedad, las personas trabajadoras independientes o informales deben ser mayorer de (18) años, no tener más de 56 años las mujeres y 61 años los hombres con edades de permanecía hasta los 57 años para las mujeres y 62 años para los hombres. Los tomadores pueden estar desempeñando cualquier trabajo o labor que le genere ingresos como trabajador independiente o informal.

El MS brinda tres opciones para tomarlo teniendo en cuenta que los niveles de ingreso de cada tomador del MS y la población a la que va dirigida, a saber, estratos 1, 2 y 3 sin que esto excluya a cualquier persona que desee tomar el seguro. Por ello, la prima mensual de este producto varía entre los 4.000 y 14.000 pesos de acuerdo con la capacidad de pago que pueda tener la persona. El pago se realiza al momento de tomar el seguro que se direcciona en canales de distribución como tiendas, supermercados, puntos de pago barriales que son de fácil acceso para esta población.

\subsection{Aspectos técnicos, administrativos y financieros}

Las tasas comerciales para la cobertura de alimentación por desempleo se calcularon teniendo en cuenta la variabilidad de las tasas de desempleo en los estratos 1, 2 y 3 y los criterios dados en la autorización de ramos de seguros por la superintendencia financiera. Se tuvieron en cuenta el 
cumplimiento de los criterios de equidad en la correlación de la prima y el riesgo (a mayor riesgo mayor prima); la suficiencia en el que la prima comercial resultante cubre razonablemente la prima de riesgo y los gastos operacionales del seguro, tales como comisiones, gastos administrativos y prevé un margen de utilidad; la homogeneidad en las características de la población objeto de estudio tanto cuantitativas como cualitativas (población ocupada formal y población ocupada informal), y representatividad donde se tienen en cuenta los conjuntos poblaciones completos, lo que permite que los resultados obtenidos sean aplicables a toda la población objeto.

Los porcentajes de cada amparo adicional corresponden al 20\% del amparo básico los cuales pueden ser usados indistintamente por los tomadores del MS. El proceso de suscripción requiere de capacitación por parte de los asesores que estarían ubicados en espacios de mercadeo masivo (supermercados, droguerías, tiendas) y se realiza solamente con el número de identificación y la opción de vinculación laboral formal, independiente o informal. Los factores de seguridad aplicados en las dos coberturas corresponden a los criterios actuariales dado el conocimiento de los riesgos al momento de la elaboración de la nota técnica. La tasa de interés técnico anual es del 4,00\%, $(\mathrm{i}=0.04)$, para la cobertura de incapacidad parcial permanente.

\section{CONCLUSIONES}

Los MS en Colombia no han tenido mayor incidencia por los niveles de riesgo que pueden acarrear para las aseguradoras. No obstante, las poblaciones de bajos recursos son un mercado potencial en la medida que pueden aportar, ahorrar y promover buenas prácticas financieras que les permita potenciar el crecimiento económico en medio de una brecha entre ricos y pobres que los excluye de todos los sectores sociales. En tal sentido, el desempleo como problema sistémico del país, es uno de los principales causantes de pobreza. La inestabilidad laboral y los bajos porcentajes de la oportunidad de vinculación laboral son problemáticas que pueden ser mitigadas externamente por productos como el MS.

Por ello, este producto de MS es una oportunidad para que las familias puedan tener una alternativa real de apoyo al sostenimiento de una de las necesidades básicas de todo ser humano. La alimentación junto con la salud y la educación son derechos que deben ser garantizados para 
la familia y que muchas veces se ve afectado por las problemáticas de desempleo en el caso de los trabajadores formales o por la enfermedad $\mathrm{o}$ accidentes en poblaciones de trabajo informal.

Las microfinanzas son una alternativa adelantada en países en vías de desarrollo para el crecimiento socioeconómico de las familias en condición de vulnerabilidad o pobreza, quienes por sus deficientes ingresos económicos, se ven excluidos del sistema financiero formal, viéndose obligados a incurrir en operaciones financieras informales, muchas veces ilegales. De ahí, que un MS familiar para alimentación sea una alternativa de apoyo para la ingesta del núcleo familiar, apalancado por el propio ahorro y las prácticas de cuidado y asegurabilidad dentro de estas poblaciones sin perder de vista la gran responsabilidad que tiene el Estado para invertir socialmente en las condiciones mínimas de la población colombiana.

En definitiva, los MS son instrumentos de carácter social que pueden contribuir al mejoramiento de las condiciones sociales de familias en alto nivel de vulnerabilidad y que por intermediación del Estado puede generar mayor impacto por el apalancamiento financiero que puede contribuir en el mercado asegurador. Por otro lado, el desafío para el uso e impacto de los MS en Colombia se enmarca en la educación económica y financiera como estrategia para el conocimiento de estos productos a los cuales pueden tener acceso y uso las personas con bajo poder adquisitivo.

\section{BIBLIOGRAFÍA}

Beltrán, G. \& Urrego, J., (2015). Microseguro para salud y alimentación (Salim): un estudio de caso en estratos 1, 2 y 3 de la ciudad de Bogotá. Revista Finnova: Investigacion e Innovación, vol. 2.

Camargo, A., Tatin-Jalerán, C. \& Furst, L.G., (2014). Colombia, Hacia un sector de seguros inclusivo y universal.

Churchill, C. \& Matul, M., (2014). Protegiendo a los pobres: Un compendio sobre microseguros.

Mccord, M.J., Ingram, M. \& Tatin-Jaleran, C., (2014). El panorama de los microseguros en América Latina y el Caribe.

Mena, B., (2004). Microcréditos: un medio efectivo para el alivio de la pobreza. $M i$ crofinanzas.

Yunus, M., (2008). Hacia un mundo sin pobreza., vol. VII, pp. 1998-2001. 